\title{
Towards using Single EEG Channel for Human Identity Verification
}

\author{
Marwa A. Elshahed \\ Physics Department, Faculty of Women for Arts \\ Sciences and Education, Ain Shams University, Cairo, Egypt
}

\begin{abstract}
Biometrics is an interesting area of research as a result of tremendous technological advances, especially in security. It is considered as an automated technology used for identification based on biological or behavioral human traits. An electroencephalogram (EEG) is the brain electrical activity signals considered as biological traits used in biometrics systems. The primary goal of this work is trying to find a single EEG channel to be used for human identification purposes. A single EEG channel recording is used for personal identity-based verification mode, which is preferred for many subjects with instant real-time system decisions. Percent residual difference (PRD) is a common quantitative measurement used to determine the human identity-based measures the distance between two signals. The proposed system sensitivity gives $100 \%$ using some single channels placed in the parietal and occipital lobes. The proposed system takes a short time in the enrolment process with an instant decision using verification mode, which is preferred with a large number of subjects. Also, using imaginary tasks is preferred for human identity verification.
\end{abstract} lobes

Keywords-Biometric; EEG; single channel; verification; brain

\section{INTRODUCTION}

Biometrics is the field of studying the biological or behavioral traits of humans which could be used for personal identification. Biological traits depend on direct measures of shapes or characteristics of biological parts in the human body, such as iris, face, DNA, ECG, etc... Behavioral traits depend on the human behavioral such as gait, signature, voice, etc.

Not all human traits could be used as a biometric, it should be characterized by uniqueness, universality, collectability, permanence, performance, circumvention, and acceptability. There are some traits changes over time, so any biometric system should be updated after some time.

A Biometric system is based on identification or verification modes. The enrolment process is used to build the system database. It was the first and main step for any system. The identification mode is considered as one to many comparisons which takes the extracted trait from the claimed person and compares it with all that stored in the database to get its identity as in Fig. 1, while in the verification mode, which is considered as one to one comparison, takes the extracted trait and ID from the claimed person then only one template from the system database is compared with the extracted one using the ID as in Fig. 2. The biometric system operates in one of the two modes. Some biometric systems are considered as a unimodal system, which depends on only one biometric trait, while others are considered as a multimodal biometric system which depends on more than one human trait.

Electroencephalogram (EEG) signals are considered as physiological biometrics which represent the brain's electrical activity. It has become an interesting area of research for human identification due to the amazing progress in using sensors and wireless networks based on Wireless Body Area Network (WBAN) [1-6]. EEG signal is classified into five waves according to the frequencies based on human activity and this is illustrated in brief in Table I, while other studies classify EEG signals to six waves, the following five waves in addition to Mu waves with an overlapping frequency range [7$11]$.

The human brain consists of four lobes illustrated in Fig. 3 by four different colors. The frontal lobe (blue) is located in front of the brain, followed by the parietal lobe (yellow) at the top of the head, then the occipital lobe (pink) at the back of the head, while the temporal lobes (green) are located on both sides of the brain above the ears. The five senses' locations are illustrated in brain lobes as in Fig. 3.

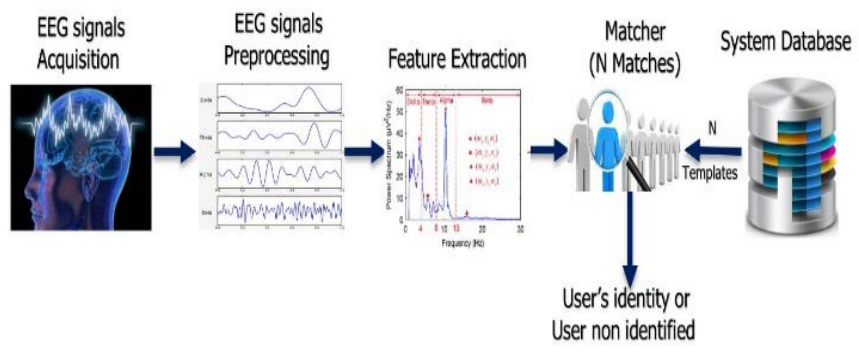

Fig. 1. Identification EEG Biometric System.

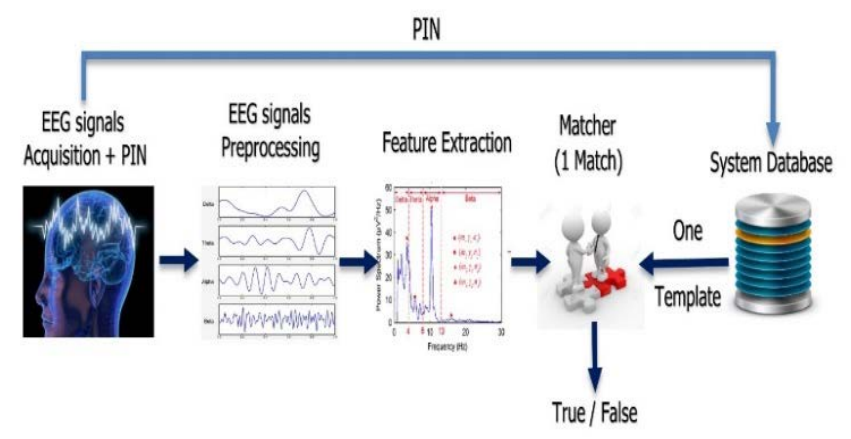

Fig. 2. Verification EEG Biometric System. 
TABLE I. EEG SIGNAL CLASSIFICATION [7].

\begin{tabular}{|l|l|l|}
\hline Name & Frequency band $(\mathbf{H z})$ & State \\
\hline $\boldsymbol{\Delta}$ WAVE & $1 \sim 3$ & Deep sleep \\
\hline $\boldsymbol{\Theta}$ WAVE & $4 \sim 7$ & Shallow sleep \\
\hline A WAVE & $8 \sim 13$ & Relaxed state \\
\hline B WAVE & $14 \sim 30$ & Active state \\
\hline $\boldsymbol{\Gamma}$ WAVE & $30 \sim$ & Mental strain \\
\hline
\end{tabular}

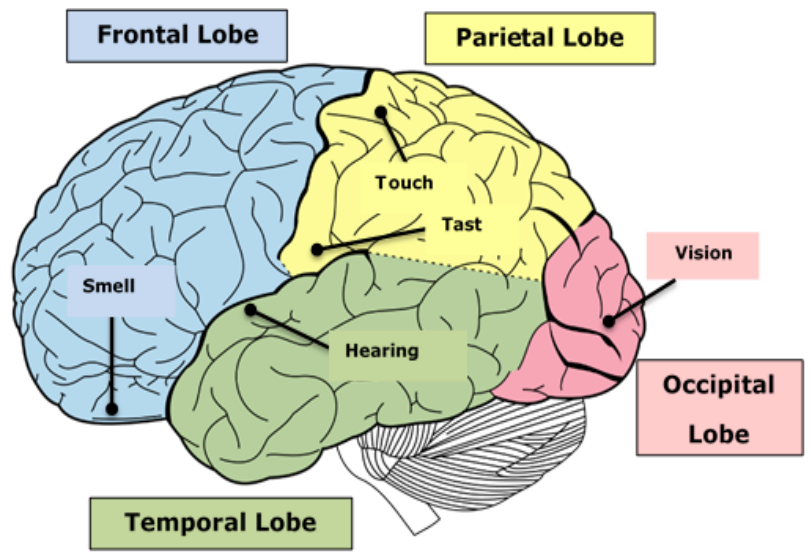

Fig. 3. The Human Brain Four Lobes.

The frontal lobe function is responsible for muscle movements, speaking, judgments, and making plans. It is described as the brain control center. Next, the parietal lobe processes information about taste and touch senses. The Occipital lobe is responsible for vision. The temporal lobe is the fourth lobe that is responsible for receiving sound from the opposite ear [12,13]. EEG signals are extracted from the human brain based on certain positions. There was an international system that illustrates these electrode or channel positions in the human scalp. This work used a database created based on the international 10-20 system as shown in Fig. 4.

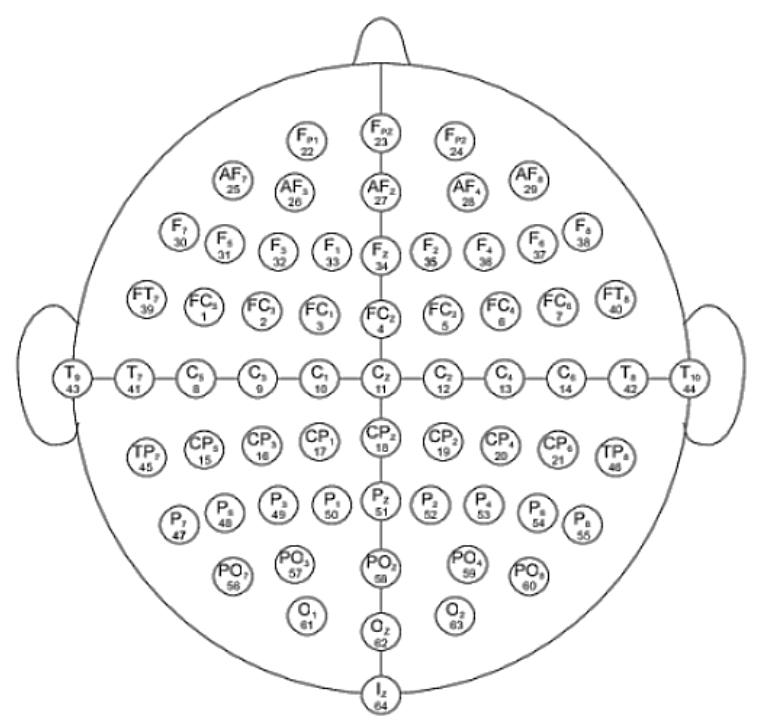

Fig. 4. 64 Electrodes Positioning based on the International 10-20 System [16].
The system depends on the relation between the cerebral cortex area and the electrode location. Also, the distances between the adjacent electrodes are equal to $10 \%$ or $20 \%$ from the whole right-left or front-back of the skill distance. The electrode name has a letter that refers to the lobe (F-frontal, Pparietal, O-O-occipital, T-temporal, C-for central positions, and Z-for midline positions) and a number that refers to the hemisphere location. The even numbers for right hemisphere positions while the odd numbers for left hemisphere positions $[14,15]$.

\section{RELATED WORK}

Authors in [17] obtained a high system accuracy rate using proposed combined Independent Component Analysis (ICA) and AR classifiers. They used only 20 subjects based on sixteen electrode placements on the scalp according to the 1020 international system. In [18], authors trying to get an EEG single channel to be used in the authentication. They used the same dataset which was used in this work based on open and closed eye states with 109 subjects, the obtained system accuracy in the range of 97-99\%. Studying to get a single EEG channel for human identification was proposed in [19] also, which is based on generating a personal identification number (PIN) from the brain activity obtained from a single active EEG channel with 3 subjects only. The high system performance is obtained using channel Cz. New features are proposed in [20] Based on a single Fp1 single channel called the concavity and convexity features in the alpha band using 23 subjects. Authors in [21] propose a new technique for EEG human verification using three channels based on Discrete Fractional Fourier Transform (DFrFT) as a feature extraction method. Their method achieves a 0.22\% Equal Error Rate (EER) with 104 subjects. In [22], an EEG biometric authentifier was proposed based on deep learning technique. Only 15 subjects were used with over 40 trials.

The experiment in [23] proposes a self-relative framework based on EEG signal for human identification using 108 subjects with closed eyes in resting state (the same dataset which was used in this work). Autoregressive is used as a feature extraction method while K-nearest neighbor is used as a classifier. They found that the openness condition gives more accurate results for identification. 19 selected channels only are used.

Most EEG biometric systems are based on identification mode. Also, they didn't test their systems for unauthorized subjects. The biometric system performance measures are neglected in calculations except the accuracy of most of them.

The objective of this research is trying to find only one EEG channel recording which could be used in a biometric system to get a personal identity with high efficiency and a short time with a large number of subjects-based verification mode.

\section{RESEARCH METHOD}

In this research, 109 subjects are used from the EEG Motor Movement/Imagery Dataset (eegmmidb) Database [16]. Table II summarizes the dataset description. The number of authorized subjects used in this work is 100 while 9 are used 
as unauthorized subjects. 64-channels were used for EEG signal recording based on the BCI2000 system [24].

Subjects performed six motor/imagery tasks. Four of them were repeated three runs, so each subject performed 14 runs. Task 1 for opening and closing left or right fist, Task 2 for imagining opening and closing left or right fist, Task 3 for opening and closing both fists or both feet, and Task 4 for imagining opening and closing both fists or both feet. Task 1 and task 3 are non-imaginary tasks, while task 2 and task 4 are imaginary tasks.

In this work, 12 runs for each subject were used for the four repeated tasks. The first and second runs of the four repeated tasks were used in the enrolment process while the third run was used to test the authorized subjects for each electrode. The Butterworth filter was applied to signals. The difference between two signals was calculated using the percent residual difference (PRD), which is a quantitative measurement:

$P R D_{n}=\sqrt{\frac{\sum_{i=1}^{M}\left(x_{0}(i)-x_{n}(i)\right)^{2}}{\sum_{j=1}^{M}\left(x_{0}(j)-\overline{x_{0}}\right)^{2}}} \times 100 \%$

Where: xo is considered as the unknown signal while $\mathrm{xn}$ is the enrolled signal for subject $n[25,26]$.

A verification model was used in this work for personal identification, which is preferred with a large number of subjects to get the system decision in a very short time. PRDth was calculated during the enrolment process for each subject. PRDn was calculated for each imposter (from the third run of tasks). A decision is obtained based on the stored database using the person's ID as illustrated in the proposed method in Fig. 5.

TABLE II. DATA SET DESCRIPTION USED IN THIS EXPERIMENT

\begin{tabular}{|l|l|}
\hline Dataset Properties & Information \\
\hline NUMBER OF SUBJECTS & 109 \\
\hline NUMBER OF CHANNELS & 64 \\
\hline SAMPLING FREQUENCY & $160 \mathrm{HZ}$ \\
\hline SIGNAL TIME LENGTH & $60 \mathrm{~s}$ \\
\hline SYSTEM & BCI2000 \\
\hline
\end{tabular}

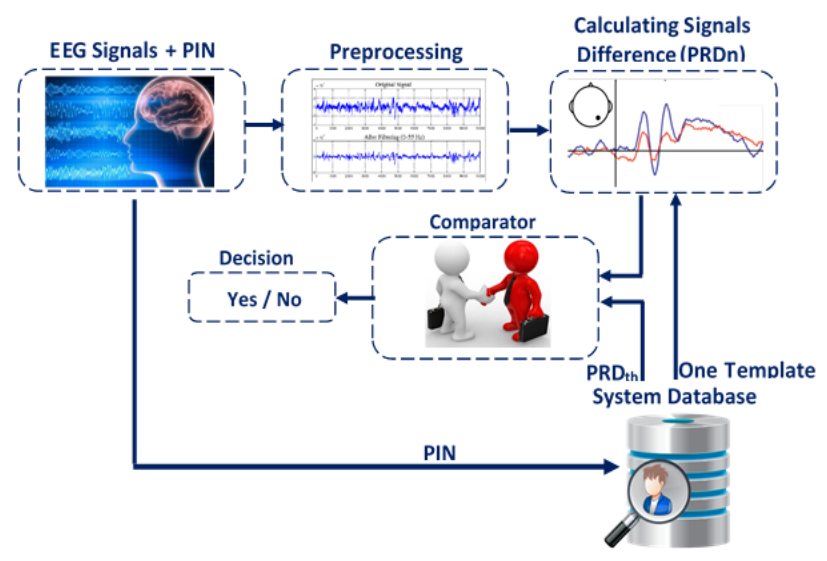

Fig. 5. The Proposed System Diagram.

\section{EXPERIMENT AND RESULTS}

In this study, all 64 channels were used and tested for authorized and unauthorized subjects using the four tasks together and with each task alone as mentioned before. The system was tested five times, namely, system-based all four tasks together, system-based task 1, system-based task 2, system-based task 3, and system-based task 4 . The system's performance is evaluated using the following measurements:

$$
\begin{aligned}
& \text { Accuracy }=\frac{T p+T n}{T p+T n+F n+F p} \times 100 \\
& F R R=\frac{F n}{T p+F n} \times 100 \\
& F A R=\frac{F p}{T n+F p} \times 100 \\
& \text { Recall }=\frac{T p}{T p+F_{N}} \times 100 \\
& \text { Percision }=\frac{T p}{T_{P}+F p} \times 100 \\
& F-\text { score }=\frac{2 \times \text { Percision } \times \text { Recall }}{\text { Percision }+ \text { Recall }}
\end{aligned}
$$

Where: Tp is true positive (Accepted knowns), Tn is true negative (rejected unknowns), $\mathrm{Fn}$ is the false negative (rejected knowns) and Fp is the false positive (accepted unknowns) [27,28].

The obtained results will be presented in detail in the following sections, starting with task 2 which gives the highest system performance.

\section{A. The Proposed System based on Task 2}

The results of the system performance based on task 2 for all 64 channels individually are shown in Table III. The results in this table are arranged in descending order according to the system accuracy. The obtained results show that the channels located in the parietal (yellow) and occipital (pink) lobes give higher system accuracy than channels located in the frontal (blue) and temporal (green) lobes. These selected colors are derived from Fig. 1. This experiment is performed using single-channel mode because the target is trying to find the best one for human identification.

Also, $\mathrm{z}$ electrodes located in the vertical center give higher system accuracy (in the parietal and occipital lobes). Four electrodes give $100 \%$ system accuracy-based task 2 (Pz, P6, CP3, and CP1). FRR reaches $0 \%$ using 12 electrodes placed in the parietal and occipital lobes and FAR reaches 0\% using 10 electrodes placed in the same lobes. Recall gives 1 using 12 electrodes while precision and f-score are given 1 using 10 electrodes distributed in the parietal and occipital lobes. The first four electrodes (Pz, P6, CP3, and CP1) in the table are given optimum system performance.

\section{B. The Proposed System based on the Four Tasks together}

The system accuracy based on the four tasks together for all 64 channels reaches $99.08 \%$ using 8 channels individually and FRR reaches $0 \%$ using 9 channels placed in the parietal and occipital lobes while FAR gives $0 \%$ using 5 electrodes in the same lobes. Recall gives 1 using 9 channels, Precision reaches to 1 using 5 channels while f score starts at $99.5 \%$. 
TABLE III. SYSTEM PERFORMANCE USING TASK 2

\begin{tabular}{|c|c|c|c|c|c|c|}
\hline Elect. & $\begin{array}{l}\text { Acc. } \\
\%\end{array}$ & FRR\% & FAR \% & Recall\% & $\begin{array}{l}\text { Per. } \\
\%\end{array}$ & $\begin{array}{l}\text { f score } \\
\%\end{array}$ \\
\hline $\mathrm{Pz}$ & 100 & 0 & 0 & 100 & 100 & 100 \\
\hline P6 & 100 & 0 & 0 & 100 & 100 & 100 \\
\hline $\mathrm{CP} 3$ & 100 & 0 & 0 & 100 & 100 & 100 \\
\hline $\mathrm{CP} 1$ & 100 & 0 & 0 & 100 & 100 & 100 \\
\hline $\mathrm{CP} 2$ & 99.08 & 1 & 0 & 99 & 100 & 99.5 \\
\hline P3 & 99.08 & 0 & 11.11 & 100 & 99.01 & 99.5 \\
\hline P2 & 99.08 & 1 & 0 & 99 & 100 & 99.5 \\
\hline P4 & 99.08 & 1 & 0 & 99 & 100 & 99.5 \\
\hline PO3 & 99.08 & 0 & 11.11 & 100 & 99.01 & 99.5 \\
\hline Poz & 99.08 & 0 & 11.11 & 100 & 99.01 & 99.5 \\
\hline $\mathrm{Oz}$ & 99.08 & 0 & 11.11 & 100 & 99.01 & 99.5 \\
\hline $\mathrm{C} 1$ & 99.08 & 0 & 11.11 & 100 & 99.01 & 99.5 \\
\hline $\mathrm{C} 2$ & 99.08 & 1 & 0 & 99 & 100 & 99.5 \\
\hline P5 & 99.08 & 0 & 11.11 & 100 & 99.01 & 99.5 \\
\hline P1 & 98.17 & 2 & 0 & 98 & 100 & 98.99 \\
\hline O1 & 98.17 & 1 & 11.11 & 99 & 99 & 99 \\
\hline $\mathrm{CPZ}$ & 98.17 & 0 & 22.22 & 100 & 98.04 & 99.01 \\
\hline $\mathrm{PO} 7$ & 98.17 & 1 & 11.11 & 99 & 99 & 99 \\
\hline P8 & 98.17 & 2 & 0 & 98 & 100 & 98.99 \\
\hline $\mathrm{PO} 4$ & 98.17 & 1 & 11.11 & 99 & 99 & 99 \\
\hline $\mathrm{C} 3$ & 98.17 & 1 & 11.11 & 99 & 99 & 99 \\
\hline $\mathrm{O} 2$ & 97.25 & 2 & 11.11 & 98 & 98.99 & 98.49 \\
\hline $\mathrm{CP} 4$ & 97.25 & 2 & 11.11 & 98 & 98.99 & 98.49 \\
\hline $\mathrm{Cz}$ & 97.25 & 0 & 33.33 & 100 & 97.09 & 98.52 \\
\hline CP5 & 96.33 & 1 & 33.33 & 99 & 97.06 & 98.02 \\
\hline C4 & 96.33 & 1 & 33.33 & 99 & 97.06 & 98.02 \\
\hline СР6 & 96.33 & 1 & 33.33 & 99 & 97.06 & 98.02 \\
\hline P7 & 96.33 & 2 & 22.22 & 98 & 98 & 98 \\
\hline PO8 & 94.5 & 5 & 11.11 & 95 & 98.96 & 96.94 \\
\hline Iz & 94.5 & 2 & 44.44 & 98 & 96.08 & 97.03 \\
\hline ТP8 & 93.58 & 6 & 11.11 & 94 & 98.95 & 96.41 \\
\hline FC2 & 93.58 & 1 & 66.67 & 99 & 94.29 & 96.59 \\
\hline FC4 & 93.58 & 3 & 44.44 & 97 & 96.04 & 96.52 \\
\hline $\mathrm{FCz}$ & 92.66 & 4 & 44.44 & 96 & 96 & 96 \\
\hline FC3 & 91.74 & 4 & 55.56 & 96 & 95.05 & 95.52 \\
\hline C6 & 90.83 & 6 & 44.44 & 94 & 95.92 & 94.95 \\
\hline C5 & 89.91 & 2 & 100 & 98 & 91.59 & 94.69 \\
\hline $\mathrm{Fz}$ & 89.91 & 4 & 77.78 & 96 & 93.2 & 94.58 \\
\hline F4 & 89.91 & 4 & 77.78 & 96 & 93.2 & 94.58 \\
\hline T9 & 88.99 & 8 & 44.44 & 92 & 95.83 & 93.88 \\
\hline TP7 & 88.99 & 7 & 55.56 & 93 & 94.9 & 93.94 \\
\hline FC1 & 88.99 & 3 & 100 & 97 & 91.51 & 94.17 \\
\hline
\end{tabular}

\begin{tabular}{|l|l|l|l|l|l|l|}
\hline Elect. & $\begin{array}{l}\text { Acc. } \\
\text { \% }\end{array}$ & FRR\% & FAR\% & Recall\% & $\begin{array}{l}\text { Per. } \\
\%\end{array}$ & $\begin{array}{l}\text { f score } \\
\%\end{array}$ \\
\hline FC6 & 88.99 & 5 & 77.78 & 95 & 93.14 & 94.06 \\
\hline F2 & 88.99 & 5 & 77.78 & 95 & 93.14 & 94.06 \\
\hline FT7 & 88.07 & 7 & 66.67 & 93 & 93.94 & 93.47 \\
\hline T7 & 88.07 & 7 & 66.67 & 93 & 93.94 & 93.47 \\
\hline F3 & 87.16 & 7 & 77.78 & 93 & 93 & 93 \\
\hline F8 & 87.16 & 6 & 88.89 & 94 & 92.16 & 93.07 \\
\hline F6 & 87.16 & 6 & 88.89 & 94 & 92.16 & 93.07 \\
\hline F5 & 86.24 & 8 & 77.78 & 92 & 92.93 & 92.46 \\
\hline FT8 & 86.24 & 7 & 88.89 & 93 & 92.08 & 92.54 \\
\hline FC5 & 85.32 & 7 & 100 & 93 & 91.18 & 92.08 \\
\hline Afz & 85.32 & 9 & 77.78 & 91 & 92.86 & 91.92 \\
\hline F1 & 85.32 & 7 & 100 & 93 & 91.18 & 92.08 \\
\hline AF4 & 85.32 & 8 & 88.89 & 92 & 92 & 92 \\
\hline F7 & 84.4 & 9 & 88.89 & 91 & 91.92 & 91.46 \\
\hline AF8 & 84.4 & 9 & 88.89 & 91 & 91.92 & 91.46 \\
\hline T8 & 84.4 & 13 & 44.44 & 87 & 95.6 & 91.1 \\
\hline AF3 & 82.57 & 12 & 77.78 & 88 & 92.63 & 90.26 \\
\hline T10 & 82.57 & 15 & 44.44 & 85 & 95.51 & 89.95 \\
\hline Fpz & 80.73 & 14 & 77.78 & 86 & 92.47 & 89.12 \\
\hline Fp2 & 80.73 & 14 & 77.78 & 86 & 92.47 & 89.12 \\
\hline Fp1 & 78.9 & 16 & 77.78 & 84 & 92.31 & 87.96 \\
\hline AF7 & 78.9 & 14 & 100 & 86 & 90.53 & 88.21 \\
\hline
\end{tabular}

\section{The Proposed System based on Task 1}

The same observations are obtained again by repeating the experiment based on task 1 individually, but the system accuracy decreases and starts at $96.33 \%$. FAR gives $0 \%$ using 3 channels. FRR is higher than that in the previous experiment. Recall starts from $98 \%$, precision gives 1 using 3 channels and f-score starts from $96 \%$, so the system performance based on the four tasks together is better than that based on task 1 alone.

\section{The Proposed System based on Task 3}

It was observed from the system performance-based task 3 for all 64 channels that $\mathrm{O} 2$ channel gives the highest system accuracy using the proposed system-based task 3 alone with 0 $\%$ FRR and $11.11 \%$ FAR. FRR is accepted using all electrodes, especially in electrodes placed in the parietal and occipital lobes than others, while FAR increases gradually and starts at $11.11 \%$. Recall gives 1 using one channel, precision reaches to $99 \%$ and $99.5 \%$ f-score.

\section{E. The Proposed System based on Task 4}

From the system performance-based task 4 for all 64 channels individually, 9 electrodes give the same system accuracy of $98.17 \%$ with $0 \%$ FAR (100\% Specificity) and $2 \%$ rejected authorized subjects only and overall, the FRR is accepted based on task4 while FAR increases in frontal and temporal electrodes. Precision reaches to 1 using 12 channels, recall reaches to $98 \%$ and f-score reaches to $98.9 \%$. 


\section{F. The Proposed System Performance Measures}

Some measures are calculated for all 64 channels using the four tasks together and with each task individually. Fig. 6 shows the average system accuracy.

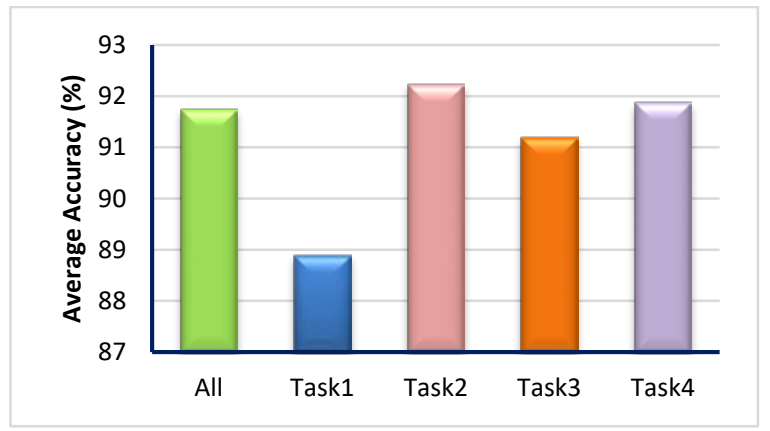

Fig. 6. The Average System Accuracy.

From the above figure, it was observed that the proposed system-based task 2 has the higher system accuracy, followed by task 4 . Task 2 and task 4 are imaginary tasks, which means that using the imaginary tasks is preferred for human authentication than non-imaginary tasks. Fig. 7 shows the average system False Reject Rate (FRR).

FRR measures the identification percentage for authorized persons who are incorrectly rejected. The proposed systembased task 3 has the best FRR is $3.8 \%$, followed by using the four tasks together is about $4 \%$ and then task 2, which is 4.45 $\%$ as shown in Fig. 7.

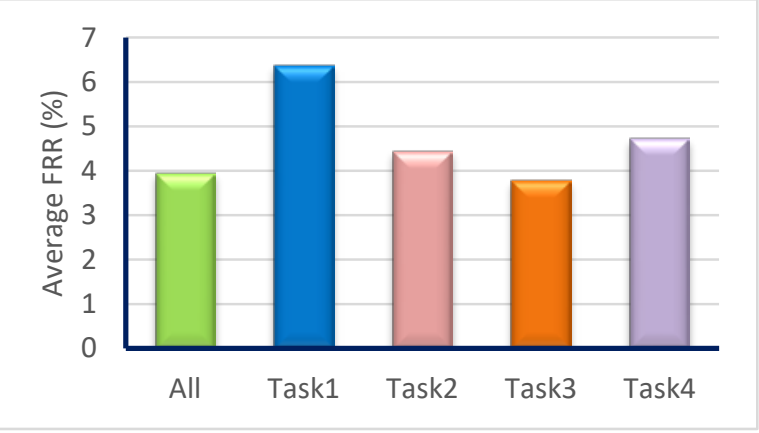

Fig. 7. The Average System FRR.

Fig. 8 shows the average system False Acceptance Rate (FAR). FAR measures the identification percentage for unauthorized persons who are incorrectly accepted.

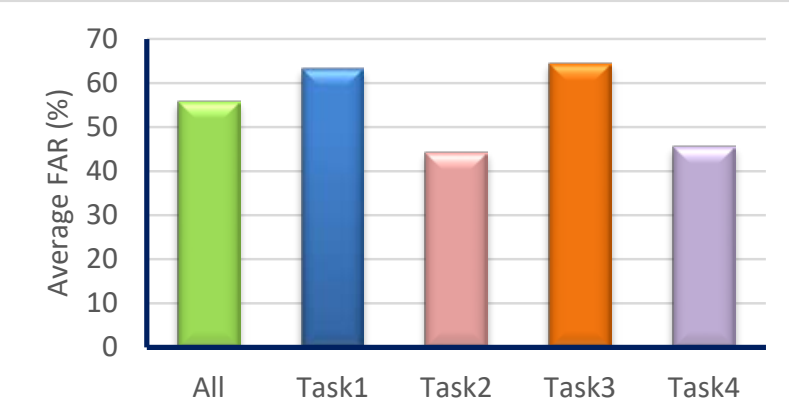

Fig. 8. The Average System FAR.
The system FAR based on task 2 is about $44.4 \%$, which is lower than others, followed by that based on task 4 , which is about $45.7 \%$. More studies will be tried to improve FAR for the proposed system.

Fig. 9 shows the average system Recall, it quantifies the number of authorized persons who are incorrectly rejected.

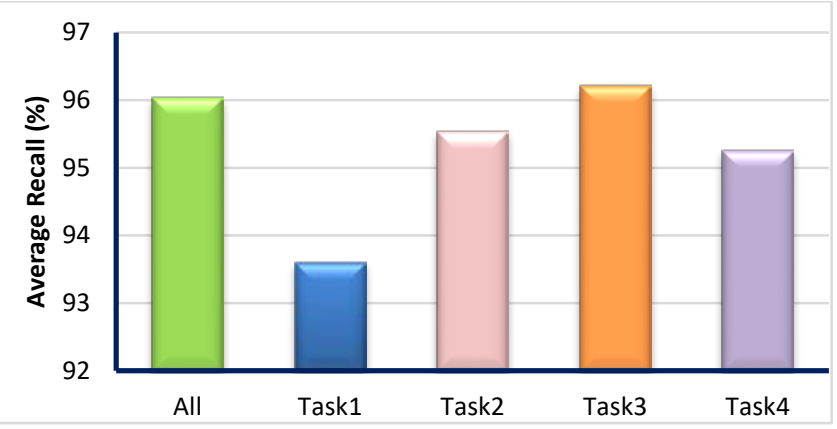

Fig. 9. The Average System Recall.

The average system recall for task 3 is about $96.2 \%$ followed by that based on all tasks together $96 \%$, then task 2 , which is equal to $95.5 \%$. Fig. 10 shows the average system precision. It quantifies the number of authorized persons who are correctly accepted.

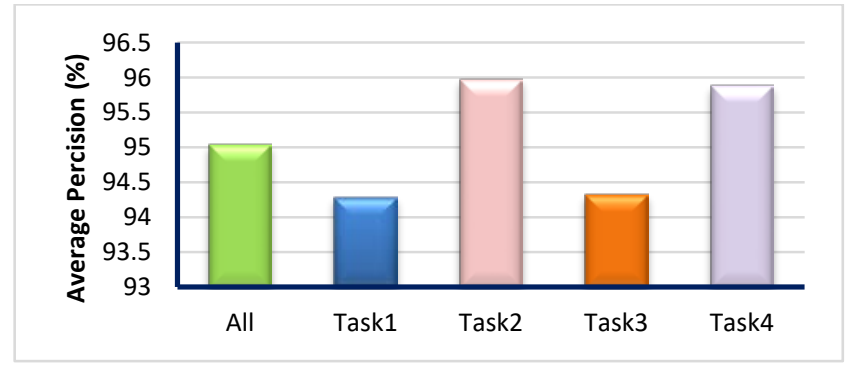

Fig. 10. The Average System Precision.

The average system precision based on task 2 is $95.98 \%$ followed by that based on task 4 which is $95.89 \%$ (approximately the same for imaginary tasks). The average system precision for all tasks together comes after the imaginary tasks, while that based on the non-imaginary tasks gives $94 \%$.

Fig. 11 shows the average system F-score. It is a single value that gives the balance between precision and recall for the proposed system.

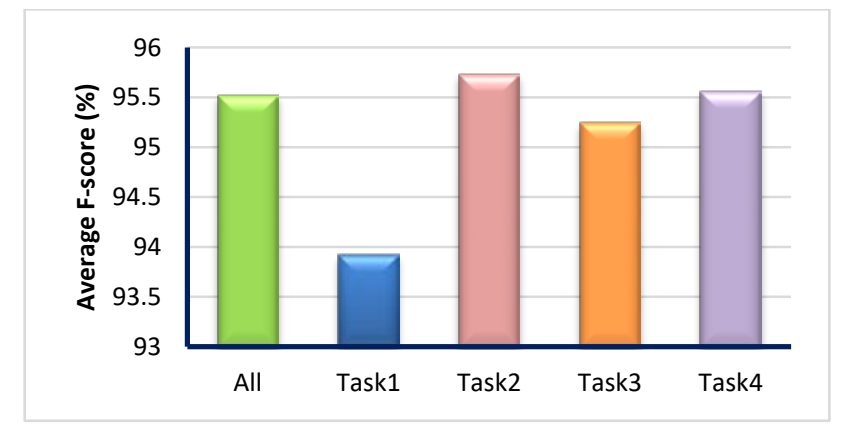

Fig. 11. The Average System F-score. 
The average system F-score based on task 2 is $95.7 \%$, which is the best score obtained by the proposed system, followed by that based on task 4 , which equals $95.5 \%$.

From this experiment, it was observed that using EEG signals collected from some electrodes placed in the parietal and occipital lobes gives a good biometric system performance for authorized and unauthorized subjects. While using electrodes placed in frontal and temporal lobes gives accepted performance for authorized subjects only. Also, not all tasks are preferred for human identification based on EEG signal. Only imaginary tasks give better system performance than non-imaginary tasks.

\section{CONCLUSION}

Biometric system-based physiological traits were more secure and difficult to mimic or penetrate. Also, using verification mode is preferred with a large number of subjects to save time. EEG signals were used for personal identity. Nowadays, it is easy to send EEG or ECG signals by network using simple sensors. So, using EEG may become a powerful tool for human identification in the next few days. Trying to find a single electrode with high efficiency is very important for using EEG as a biometric trait to become easy to use and fast to make a decision. This work is trying to find this electrode by using existing data. This data presents EEG signals obtained by performing four tasks (two of them imaginary tasks and the others are non-imaginary tasks). This work-based on PRD, which is a simple measurement used to measure the difference between two signals. The proposed system was repeated for five experiments. The first one is based on using the four tasks together to make the system decisions, the second is based on task 1 only, the third is based on task 2, the fourth is based on task 3 while the fifth is based on task 4 . Tasks 1 and 3 are non-imaginary tasks, while tasks 2 and 4 are imaginary.

The observations from the obtained results show that the proposed system-based task 2 is better than using other tasks individually and the four tasks together, the system performance-based task 4 is better than that based on task 1 and task 3 individually, which means that using the imaginary tasks is more suitable than using non-imaginary tasks for human authentication. Also, using electrodes placed in the parietal and occipital lobes is better than those placed in other lobes, and electrodes near the vertical brain center give higher system performance. Pz, P6, CP3, and CP1 electrodes give $100 \%$ system accuracy-based task 2 individually. It was observed that the proposed system sensitivity (recognize authorized subjects) is good and acceptable using all electrodes and increases as mentioned before in the parietal and occipital lobes electrodes, while the proposed system specificity (recognize unauthorized subjects) is acceptable in some electrodes placed in parietal and occipital lobes only, so more studies needed to improve the proposed system specificity. The proposed system gives instantaneous decision because its construction is based on verification mode, which makes it very suitable for large numbers.

\section{REFERENCES}

[1] Marwa A. Elshahed,” Personal identity verification based ECG biometric using non-fiducial features", International Journal of Electrical and Computer Engineering (IJECE), ISSN: 2088-8708, DOI: 10.11591/ijece.v10i3.pp3007-3013,2020.

[2] Mohamad El-Abed and Christophe Charrier, "Evaluation of Biometric Systems”, New Trends and Developments in Biometrics, pp. 149 - 169, ff10.5772/52084ff. ffhal-00990617f, 2012.

[3] A.S. Raju and V. Udayashankara," Performance Evaluation of Multimodal Biometrics System”, International Journal of Pure and Applied Mathematics, Volume 118 No. 5, 367-382,2018.

[4] Abdullah Alhayajneh, Alessandro N. Baccarini, Gary M. Weiss, Thaier Hayajneh and Aydin Farajidavar," Biometric Authentication and Verification for Medical Cyber Physical Systems",MDPI, Electronics 7(12), 436, 2018.

[5] K. P. Thomas and A. P. Vinod, "Utilizing individual alpha frequency and delta band power in EEG based biometric recognition,” in 2016 IEEE International Conference on Systems, Man, and Cybernetics (SMC), pp. 004787-00479, 2016.

[6] Q. Gui, Z. Jin, and W. Xu, "Exploring EEG-based biometrics for user identification and authentication,” 2014 IEEE Signal Process. Med. Biol. Symp. IEEE SPMB 2014 - Proc., 2015.

[7] Masahiro Hakodaa, Hirokazu Miurab , Noriyuki Matsudab , Fumitaka Uchiob and Hirokazu Takib , "Measurement of Brain Activity on Force Adjustment Skill Acquisition by using EEG”, International Conference on Knowledge Based and Intelligent Information and Engineering Systems, KES2017, 6-8 September Marseille, France.2017.

[8] https://www.sciencedirect.com/topics/biochemistry-genetics-andmolecular-biology/electroencephalogram.

[9] A. Elakkiya, S.Ramkumar, G. Emayavaramban and P.Buvaneswari,” A Survey of Biometrics Person Identification System Using EEG Brain Signal”, International Journal of Pure and Applied Mathematics, Volume 119, No. 15 2018, 3471-3475, ISSN: 1314-3395,2018.

[10] S. Siuly, Y. Li, Y. Zhang, EEG signal analysis and classification, (New York, NY: Springer Berlin Heidelberg), ISBN 9783319476520, 2017.

[11] Zuzana Koudelková1 and Martin Strmiska,” Introduction to the identification of brain waves based on their frequency", MATEC Web of Conferences 210, $05012,2018$.

[12] Jiawei Zhang," Secrets of the Brain: An Introduction to the Brain Anatomical Structure and Biological Function”, IFM LAB TUTORIAL SERIES \# 4,2019.

[13] https://www.md-health.com/Lobes-Of-The-Brain.html.

[14] Margitta Seeck, Laurent Koessler, Thomas Bast, Frans Leijten, Christoph Michel, Christoph Baumgartner, Bin He and Sándor Beniczky,” The standardized EEG electrode array of the IFCN”, Clinical Neurophysiology journal,2017.

[15] International 10-20 system manual, Trans Cranial Technologies ldt, 2012.

[16] https://archive.physionet.org/physiobank/database/eegmmidb/.

[17] Chesada Kaewwit, Chidchanok Lursinsap and Peraphon Sophatsathit,” High Accuracy EEG Biometrics Identification using ICA And AR Model”, Journal of ICT, 16, pp: 354-373, (Dec) 2017.

[18] R. Suppiah and A. P. Vinod, "Biometric identification using single channel EEG during relaxed resting state," in IET Biometrics, vol. 7, no. 4, pp. 342-348, 72018.

[19] Ramaswamy Palaniappan, Jenish Gosalia, Kenneth Revett and Andrews Samraj,” PIN Generation Using Single Channel EEG Biometric”, Springer, ACC 2011, Part IV, CCIS 193, pp. 378-385, 2011.

[20] Isao Nakanishi, Sadanao Baba and Chisei Miyamoto,"EEG Based Biometric Authentication Using New Spectral Features”,Proc. of 2009 IEEE International Symposium on Intelligent Signal Processing and Communication Systems (ISPACS2009), Dec 2009. 
[21] Sarineh Keshishzadeh, Ali Fallah and Saeid Rashidi,” Electroencephalogram Based Biometrics: A Fractional Fourier Transform Approach”, ICBEA '18, ACM ISBN 978-1-4503-63945/18/05,2018.

[22] JISSY J, RESHMA V K,” Brain Fastener as Eeg Biometric Authentifier", International Research Journal of Engineering and Technology (IRJET), Volume: 07, Issue: 06 , June 2020.

[23] 23 Meriem Romaissa Boubakeur and Guoyin Wang,” Self-Relative Evaluation Framework for EEG-Based Biometric Systems”, MDPI, Sensors 2021, 21, 2097, 2021.

[24] http://www.bci2000.org

[25] Chan, Adrian D.C.; Hamdy, Mohyledin M.; Badre, Armin and Badee, Vesal," wavelet distance measure for person identification using electrocardiograms”, IEEE Transactions on Instrumentation and Measurement · February 2008.

[26] Ruqaiya Khanam and Syed Naseem Ahmad,” Selection of Wavelets for Evaluating SNR, PRD and CR of ECG Signal”, International Journal of Engineering Science and Innovative Technology (IJESIT) Volume 2, Issue 1, January 2013.

[27] Kareem Hatam Nahavandi,” Calculating Sensitivity, Specificity and Predictive Values for MedicalDiagnostic Tests”, Gene Cell Tissue, doi: 10.5812/gct.80270,2018.

[28] Mahsa Zeynali and Hadi Seyedarabi,” EEG-based single-channel authentication systems with optimum electrode placement for different mental activities”, biomedical journal 42 ( 2019 ) 261 -2 6 7, 2019. 